\title{
A Chiral Bis-Sulfoxide Ligand in Late-Transition Metal Catalysis; Rhodium- Catalyzed Asymmetric Addition of Arylboronic Acids to Electron-Deficient
}

\section{Olefins.}

\author{
Ronaldo Mariz, Xinjun Luan, Michele Gatti, Anthony Linden, and Reto Dorta* \\ Institute of Organic Chemistry, University of Zurich, Winterthurerstrasse 190, \\ CH-8057, Zurich, Switzerland. \\ Email:dorta@oci.uzh.ch
}

\section{Supporting information}

\section{General:}

All reactions were carried out using standard Schlenk or glovebox (Mecaplex or Innovative Technology) techniques under nitrogen. NMR spectra were collected on an AV2 $400 \mathrm{MHz}$ Bruker spectrometer. HR-MS was acquired on a Finnigan MAT 95 (Finnigan MAT95, San Jose, CA; USA) double-focusing magnetic sector mass spectrometer (geometry BE) and GC/MS analysis was done on a Finnigan Voyager GC8000 Top. Elemental analysis was done on a Leco CHN-932 analyzer. $[\alpha]_{\mathrm{D}}$ values were measured on a Jasco P-2000 Polarimeter using a filtered Hg lamp (effective wave length $=589 \mathrm{~nm}$ ). X-ray crystallography was performed on a Nonius KappaCCD area-detector diffractometer using graphite-monochromated Mo $K \alpha$ radiation $(\lambda=$ $0.71073 \AA$ ) and an Oxford Cryosystems Cryostream 700 cooler. All known compounds were according to literature data. ${ }^{1-4}$ 


\section{Materials:}

Solvents were purchased in the best quality available, degassed by purging thoroughly with nitrogen and dried over activated molecular sieves of appropriate size. Alternatively, they were purged with argon and passed through alumina columns in a solvent purification system (Innovative Technology). 2,2'-Dibromo-1,1'-binaphthyl was purchased from Strem, n-BuLi, $(1 S, 2 R, 5 S)-(+)-M e n t h y l-(R)-p$-toluenesulfinate, $\quad(1 R, 2 S, 5 R)-(-)-M e n t h y l-(S)-p$-toluenesulfinate, substrates 3a-d and boronic acids 4l-z were purchased from Acros, Aldrich or Fluka and used as received except for 3c, which was flash chromatographed (Silica gel, Hexane/Et ${ }_{2} \mathrm{O}$ 19:1) prior to use. Solvents for NMR spectroscopy were degassed with nitrogen and dried over molecular sieves. Racemic 6-Methyl-2-cyclohexen-1-one 3e was synthesized following a literature procedure. $^{5}$

Synthesis of ligands and metal precursors:

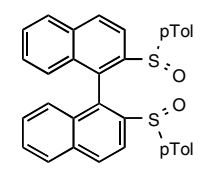

$\left(M, R_{S}, R_{S}\right) \quad$ and $\quad\left(P, R_{S}, R_{S}\right) \quad 1,1^{\prime}$-Binaphthalene-2,2'-diyl-bis-(p-tolylsulfoxide), $p$-tolBINASO (1): To a solution of 2,2'-Dibromo-1,1'-binaphthalyl (4.00 g, $9.71 \mathrm{mmol})$ and TMEDA (3.22 $\mathrm{ml}, 21.35 \mathrm{mmol})$ in dry THF (60 mL) was added dropwise n-BuLi (13.35 ml, $21.35 \mathrm{mmol}$, 1.6 $\mathrm{M}$ in hexanes) at $-78^{\circ} \mathrm{C}$, under nitrogen. The reaction mixture was stirred for 45 minutes after which a solution of $(1 S, 2 R, 5 S)-(+)-M e n t h y l(R)-p$-toluenesulfinate $(6.29 \mathrm{~g}, 21.35 \mathrm{mmol})$ in THF (60 ml) was added dropwise. After 1 hour at $-78^{\circ} \mathrm{C}$ the reaction was allowed to warm to $0^{\circ} \mathrm{C}$ and stirring was kept for one more hour at this temperature and an addition hour at $25^{\circ} \mathrm{C}$. The resulting mixture was quenched with water $(100 \mathrm{ml})$, the organic phase separated and the water was extracted with $\mathrm{CH}_{2} \mathrm{Cl}_{2}(3 \times 30 \mathrm{ml})$. The combined organic layer was dried with $\mathrm{MgSO}_{4}$, and concentrated to a small volume. Flash chromatography of the crude material with 
Hexanes/EtOAc (4:1 to 1:1) afforded the two diastereoisomers of $p$-tol-BINASO (3.71 g, $72 \%$ overall).

$\left(\boldsymbol{M}, \boldsymbol{R}_{S}, \boldsymbol{R}_{S}\right)$-p-tol-BINASO $(1.74 \mathrm{~g}, 33.8 \%$ yield, First diastereoisomer eluted from flash chromatography) $-{ }^{1} \mathrm{H}-\mathrm{NMR}\left(400 \mathrm{MHz}, \mathrm{CDCl}_{3}\right): \delta=2.32(\mathrm{~s}, 6 \mathrm{H}), 7.09-7.15(\mathrm{~d}, J=8.5 \mathrm{~Hz}, 4 \mathrm{H})$, 7.18-7.23 (d, $J=8.3 \mathrm{~Hz}, 4 \mathrm{H}), 7.27-7.32(\mathrm{~d}, J=9.4 \mathrm{~Hz}, 2 \mathrm{H}), 7.39-7.46(\mathrm{t}, J=9.8 \mathrm{~Hz}, 2 \mathrm{H}), 7.55-$ $7.59(\mathrm{~d}, J=8.7 \mathrm{~Hz}, 2 \mathrm{H}), 7.59-7.64(\mathrm{t}, J=8.2 \mathrm{~Hz}, 2 \mathrm{H}), 7.92-7.97(\mathrm{~d}, J=8.2 \mathrm{~Hz}, 2 \mathrm{H}), 8.07-8.12$ $(\mathrm{d}, J=8.4 \mathrm{~Hz}, 2 \mathrm{H}) \mathrm{ppm} .{ }^{13} \mathrm{C}-\mathrm{NMR}\left(400 \mathrm{MHz}, \mathrm{CDCl}_{3}\right): \delta=21.45$ (s), 122.86 (s), 125.93 (s), 127.73 (s), 128.35 (d, J = $7.0 \mathrm{~Hz}), 128.96$ (s), 129.87 (s), 131.59 (s), 132.91 (s), 134.86 (s), $136.52(\mathrm{~s}), 140.35$ (s), $141.16(\mathrm{~s}), 142.15(\mathrm{~s}) \mathrm{ppm} .[\alpha]_{\mathrm{D}}{ }^{25}=+7.54\left(\mathrm{c}=1.0, \mathrm{CHCl}_{3}\right) . \mathrm{HRMS}$ calculated for $\mathrm{C}_{34} \mathrm{H}_{26} \mathrm{O}_{2} \mathrm{~S}_{2}=530.137$, found $553.127\left(+\mathrm{Na}^{+}\right)$. A solution in $\mathrm{CHCl}_{3}$ layered with a mixture of Hexanes/EtOAc 3:1 afforded colorless crystals suitable for X-ray analysis (see CIF file).

$\left(\boldsymbol{P}, \boldsymbol{R}_{S}, \boldsymbol{R}_{S}\right)$-p-tol-BINASO $(1.97 \mathrm{~g}, 38.2 \%$ yield, second diastereoisomer eluted from flash chromatography) $-{ }^{1} \mathrm{H}-\mathrm{NMR}\left(400 \mathrm{MHz}, \mathrm{CDCl}_{3}\right): \delta=2.04(\mathrm{~s}, 6 \mathrm{H}), 6.13-6.17(\mathrm{~d}, J=9.1 \mathrm{~Hz}, 2 \mathrm{H})$, 6.53-6.57 (d, $J=8.1 \mathrm{~Hz}, 4 \mathrm{H}), 6.60-6.65(\mathrm{~d}, J=8.1 \mathrm{~Hz}, 4 \mathrm{H}), 6.72-6.78(\mathrm{t}, J=8.3 \mathrm{~Hz}, 2 \mathrm{H}), 7.33-$ $7.39(\mathrm{~d}, J=9.3 \mathrm{~Hz}, 2 \mathrm{H}), 7.86-7.90(\mathrm{~d}, J=8.2 \mathrm{~Hz}, 2 \mathrm{H}), 8.26-8.30(\mathrm{~d}, J=8.7 \mathrm{~Hz}, 2 \mathrm{H}), 8.55-8.59$ $(\mathrm{d}, J=8.8 \mathrm{~Hz}, 2 \mathrm{H}) \mathrm{ppm} .{ }^{13} \mathrm{C}-\mathrm{NMR}\left(400 \mathrm{MHz}, \mathrm{CDCl}_{3}\right): \delta=21.29$ (s), 119.77 (s), 125.79 (s), 126.20 (s), 127.03 (d, $J=77.9 \mathrm{~Hz}), 128.28$ (s), 129.23 (s), 130.19 (s), 130.88 (s), 132.39 (s), 134.21 (s), 140.69 (s), 141.69 (s), 142.76 (s) ppm. $[\alpha]_{\mathrm{D}}^{25}=+3.80\left(\mathrm{c}=1.0, \mathrm{CHCl}_{3}\right) . \mathrm{HRMS}$ calculated for $\mathrm{C}_{34} \mathrm{H}_{26} \mathrm{O}_{2} \mathrm{~S}_{2}=530.137$, found $553.126\left(+\mathrm{Na}^{+}\right)$. Obtained as white foam after high vacuum.

Using (1R,2S,5R)-(-)-Menthyl-(S)-p-toluenesulfinate and following the same procedure, were also synthesized the pair of diastereoismers $\left(M, S_{S}, S_{S}\right)$-p-tol-BINASO and $\left(P, S_{S}, S_{S}\right)$ - $p$-tolBINASO, and were obtained in similar yields. Analytical data according to the literature. ${ }^{6}$ 


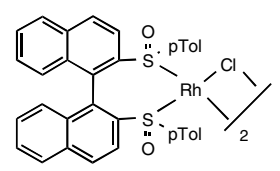

$\left[\left\{\left(\boldsymbol{P}, \boldsymbol{R}_{S}, \boldsymbol{R}_{S}\right)-\boldsymbol{p} \text {-tol-BINASO }\right\} \mathbf{R h C l}\right]_{2}(\mathbf{2}):$ To a solution of $\left[\left(\mathrm{C}_{2} \mathrm{H}_{4}\right)_{2} \mathrm{RhCl}\right]_{2}(208.0 \mathrm{mg}, 0.535$ mmol) in $\mathrm{CH}_{2} \mathrm{Cl}_{2}(30 \mathrm{ml})$ was added a solution of $\left(P, R_{S}, R_{S}\right)$-p-tol-BINASO $(567.7 \mathrm{mg}, 1.07$ mmol $)$ in $\mathrm{CH}_{2} \mathrm{Cl}_{2}(60 \mathrm{ml})$ in a $250 \mathrm{ml}$ Schlenk tube inside the glovebox. The resulting solution was stirred for 15 hours at room temperature with the valve of the tube slightly open to release ethylene. The solution is concentrated to a small volume $(\sim 3 \mathrm{ml})$, filtered through celite, layered with THF $(50 \mathrm{ml})$ and let for 24 hours at $-35^{\circ} \mathrm{C}$. The burgundy crystals are then decanted, washed with THF $(2 \times 10 \mathrm{ml})$ and dried under high vacuum to afford $681 \mathrm{mg}$ of $\left[\left\{\left(P, R_{S}, R_{S}\right)-p\right.\right.$ tol-BINASO $\} \mathrm{RhCl}_{2}$ (95.2\% yield). ${ }^{1} \mathrm{H}-\mathrm{NMR}\left(400 \mathrm{MHz}, \mathrm{CD}_{2} \mathrm{Cl}_{2}\right): \delta=1.95(\mathrm{~s}, 6 \mathrm{H}), 6.35-6.41$ $(\mathrm{d}, J=8.5 \mathrm{~Hz}, 2 \mathrm{H}), 6.51-6.57(\mathrm{~d}, J=7.9 \mathrm{~Hz}, 4 \mathrm{H}), 6.95-7.03(\mathrm{t}, J=7.2 \mathrm{~Hz}, 2 \mathrm{H}), 7.35-7.43(\mathrm{t}, J=$ $7.3 \mathrm{~Hz}, 2 \mathrm{H}), 7.71-7.77(\mathrm{~d}, J=8.2 \mathrm{~Hz}, 2 \mathrm{H}), 7.79(\mathrm{br}, 4 \mathrm{H}), 8.08-8.14(\mathrm{~d}, J=8.8 \mathrm{~Hz}, 2 \mathrm{H}), 8.47-$ $8.53(\mathrm{~d}, J=8.9 \mathrm{~Hz}, 2 \mathrm{H}) \mathrm{ppm} .{ }^{13} \mathrm{C}-\mathrm{NMR}\left(400 \mathrm{MHz}, \mathrm{CD}_{2} \mathrm{Cl}_{2}\right): \delta=21.37$ (s), 121.86 (s), 127.23 (s), 127.56 (br), 127.65 (s), 127.96 (s), 128.06 (s), 128.64 (s), 129.29 (s), 130.83 (s), 131.97 (s), 134.95 (s), 140.99 (s), 142.17 (s), 145.31 (s) ppm. Elemental analysis: calculated C = 61.04\%, H $=3.92 \%$; found $\mathrm{C}=60.15 \%$ and $\mathrm{H}=4.14 \%$.

Following the same procedure $\left[\left\{\left(M, S_{S}, S_{S}\right) \text {-p-tol-BINASO }\right\} \mathrm{RhCl}\right]_{2}$ was also synthesized in similar yield.

\section{Catalysis, general remarks:}

The racemic products of all of the reactions shown below have also been obtained for comparison of HPLC results.

Optimization of the 1,4-addition of phenylboronic acid to 2-cyclohexen-1-one:

\begin{tabular}{c|c|c|c|c|c|c|c|c}
\hline Entry & $\begin{array}{c}\text { Catalyst } \\
(\mathbf{m o l} \mathbf{R h} \%)\end{array}$ & $\begin{array}{c}\text { Eq. } \\
\mathbf{P h B}(\mathbf{O H})_{\mathbf{2}}\end{array}$ & Base/Eq. & $\begin{array}{c}\text { Solvent/ } \\
\mathbf{H}_{\mathbf{2}} \mathbf{O}(\mathbf{1 0 : 1})\end{array}$ & $\begin{array}{c}\text { Temp. } \\
\left({ }^{\circ} \mathbf{C}\right)\end{array}$ & $\begin{array}{c}\text { Time } \\
(\mathbf{h})\end{array}$ & $\begin{array}{c}\text { Yield } \\
(\boldsymbol{\%})\end{array}$ & $\begin{array}{c}\text { e.e } \\
(\boldsymbol{\%})\end{array}$ \\
\hline 1 & 2.5 & 2 & $\mathrm{KOH} / 1$ & Dioxane & $25^{\circ} \mathrm{C}$ & 5 & 16 & $>98$ \\
\hline 2 & 2.5 & 2 & $\mathrm{KOH} / 0.5$ & Dioxane & $25^{\circ} \mathrm{C}$ & 5 & 22 & $>98$ \\
\hline 3 & 2.5 & 2 & $\mathrm{KOH} / 0.25$ & Dioxane & $25^{\circ} \mathrm{C}$ & 5 & 20 & $>98$ \\
\hline 4 & 2.5 & 1.1 & $\mathrm{KOH} / 0.5$ & Dioxane & $25^{\circ} \mathrm{C}$ & 5 & 10 & $>98$ \\
\hline 5 & 2.5 & 3 & $\mathrm{KOH} / 0.5$ & Dioxane & $25^{\circ} \mathrm{C}$ & 5 & 30 & $>98$ \\
\hline
\end{tabular}




\begin{tabular}{c|c|c|c|c|c|c|c|c}
\hline 6 & 2.5 & 4 & $\mathrm{KOH} / 0.5$ & Dioxane & $25^{\circ} \mathrm{C}$ & 5 & 35 & $>98$ \\
\hline 7 & 5.0 & 3 & $\mathrm{KOH} / 0.5$ & Dioxane & $25^{\circ} \mathrm{C}$ & 5 & 28 & $>98$ \\
\hline 8 & 10.0 & 3 & $\mathrm{KOH} / 0.5$ & Dioxane & $25^{\circ} \mathrm{C}$ & 5 & 31 & $>98$ \\
\hline 9 & 2.5 & 2 & $\mathrm{KOH} / 0.5$ & Dioxane & $40^{\circ} \mathrm{C}$ & 5 & 21 & $>98$ \\
\hline 10 & 2.5 & 2 & $\mathrm{KOH} / 0.5$ & Dioxane & $60^{\circ} \mathrm{C}$ & 5 & 23 & $>98$ \\
\hline 11 & 2.5 & 2 & $\mathrm{KOH} / 0.5$ & Dioxane & $80^{\circ} \mathrm{C}$ & 5 & 24 & $>90$ \\
\hline 12 & 2.5 & 2 & $\mathrm{KOH} / 0.5$ & Dioxane & $100^{\circ} \mathrm{C}$ & 5 & 14 & $>86$ \\
\hline 13 & 2.5 & 2 & $\mathrm{KOH} / 0.5$ & Toluene & $40^{\circ} \mathrm{C}$ & 0.5 & 99 & $>98$ \\
\hline 14 & 2.5 & 1.1 & $\mathrm{KOH} / 0.5$ & Toluene & $40^{\circ} \mathrm{C}$ & 0.5 & 99 & $>98$ \\
\hline 15 & 1.0 & 2 & $\mathrm{KOH} / 0.5$ & Toluene & $40^{\circ} \mathrm{C}$ & 2 & 96 & $>98$ \\
\hline 16 & 2.5 & 2 & $\mathrm{KOH} / 0.5$ & Toluene & $25^{\circ} \mathrm{C}$ & 0.5 & 95 & $>98$ \\
\hline 17 & 1.5 & 1.1 & $\mathrm{KOH} / 0.5$ & Toluene & $40^{\circ} \mathrm{C}$ & 1 & 99 & $>98$ \\
\hline
\end{tabular}

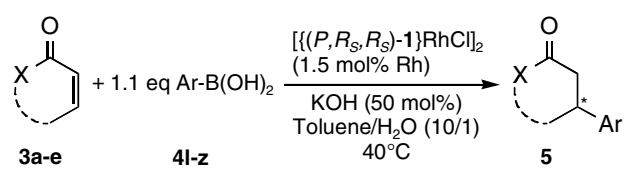

\section{General procedure for the 1,4-addition of boronic acids to enones/esters:}

In a $20 \mathrm{ml}$ vial, inside a glove box, was added the precatalyst $2(15.1 \mathrm{mg}, 0.0113 \mathrm{mmol})$ followed by $3 \mathrm{ml}$ of toluene and arylboronic acid $(1.65 \mathrm{mmol})$. The vial was fitted with a magnetic stirring bar, closed with a teflon cap, and taken out of the glove box. The degassed enone/ester $(1.5 \mathrm{mmol})$ was then added via syringe followed by degassed $\mathrm{KOH}_{\mathrm{aq}}$. $\left(2.5 \mathrm{M}\right.$ in $\mathrm{H}_{2} \mathrm{O}$, $0.3 \mathrm{ml}, 0.75 \mathrm{mmol}$ ). The reaction was stirred at $40^{\circ} \mathrm{C}$ for the appropriate time (followed by GCMS until completion). The reaction mixture was directly charged onto a column (silica gel) and flash chromatographed with a mixture of hexane/ $\mathrm{Et}_{2} \mathrm{O}$ or hexane/EtOAc to afford the product.

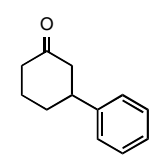

3-Phenylcyclohexanone (5al): Eluted with Hexane/Et ${ }_{2} \mathrm{O}$ 9:1, obtained as colorless oil (258.7 mg, 99\% yield). HPLC: 98\% ee, Chiralcel OD-H column (n-hexane/2-propanol, 98:2, 0.5 $\mathrm{mL} / \mathrm{min}$ ); $t_{\mathrm{R}}: 24.3 \mathrm{~min}$ (major), $26.6 \mathrm{~min}$ (minor) for reaction with $\left[\left(\left(P, R_{S}, R_{S}\right)\right.\right.$ - $p$-tolBINASO)RhCl $]_{2}$ and $t_{\mathrm{R}}: 24.3 \mathrm{~min}$ (minor), $26.6 \mathrm{~min}$ (major) for reaction with $\left[\left(\left(M, S_{S}, S_{S}\right)\right.\right.$ - $p$-tolBINASO)RhCl $]_{2} .{ }^{1} \mathrm{H}-\mathrm{NMR}\left(400 \mathrm{MHz}, \mathrm{CDCl}_{3}\right): \delta=1.76-1.96(\mathrm{~m}, 2 \mathrm{H}), 2.10-2.24(\mathrm{~m}, 2 \mathrm{H}), 2.37-$ $2.68(\mathrm{~m}, 4 \mathrm{H}), 3.00-3.12(\mathrm{~m}, 1 \mathrm{H}), 7.24-7.31(\mathrm{~m}, 3 \mathrm{H}), 7.34-7.41(\mathrm{~m}, 2 \mathrm{H}) \mathrm{ppm} .{ }^{13} \mathrm{C}-\mathrm{NMR}(400$ 
$\mathrm{MHz}, \mathrm{CDCl}_{3}$ ): $\delta=25.74$ (s), 32.99 (s), 41.39 (s), 44.95 (s), 49.15 (s), 126.77 (s), 126.89 (s), $128.89(\mathrm{~s}), 144.56(\mathrm{~s}), 211.17$ (s) ppm.

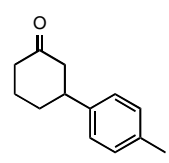

3-(4-Methylphenyl)cyclohexanone (5am): Eluted with $\mathrm{Hexane} / \mathrm{Et}_{2} \mathrm{O}$ 9:1, obtained as white solid (273.9 mg, 97\% yield). HPLC: 96\% ee, Chiralcel OD-H column (n-hexane/2-propanol, 99.9:0.1, $0.5 \mathrm{~mL} / \mathrm{min}$ ); $t_{\mathrm{R}}: 113.0 \mathrm{~min}$ (major), $135.3 \mathrm{~min}$ (minor). ${ }^{1} \mathrm{H}-\mathrm{NMR}\left(400 \mathrm{MHz}, \mathrm{CDCl}_{3}\right): \delta$ $=1.70-1.90(\mathrm{~m}, 2 \mathrm{H}), 2.03-2.19(\mathrm{~m}, 2 \mathrm{H}), 2.33(\mathrm{~s}, 3 \mathrm{H}), 2.35-2.62(\mathrm{~m}, 4 \mathrm{H}), 2.92-3.03(\mathrm{~m}, 1 \mathrm{H})$, 7.08-7.18 (m, 4H) ppm. ${ }^{13} \mathrm{C}-\mathrm{NMR}\left(400 \mathrm{MHz}, \mathrm{CDCl}_{3}\right): \delta=21.19$ (s), 25.77 (s), 33.13 (s), 41.42 (s), 44.61 (s), 49.30 (s), 126.66 (s), 129.56 (s), 136.48 (s), 141.66 (s), 211.34 (s) ppm.

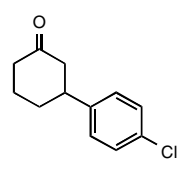

3-(4-Chlorophenyl)cyclohexanone (5an): Eluted with $\mathrm{Hexane}_{2} \mathrm{Et}_{2} \mathrm{O}$ 9:1, obtained as white solid (269.2 mg, 86\% yield). HPLC: $98 \%$ ee, Chiralcel OJ-H column (n-hexane/2-propanol, 99:1, $1.0 \mathrm{~mL} / \mathrm{min}$ ); $t_{\mathrm{R}}: 25.3 \mathrm{~min}$ (major), $29.3 \mathrm{~min}$ (minor). ${ }^{1} \mathrm{H}-\mathrm{NMR}\left(400 \mathrm{MHz}, \mathrm{CDCl}_{3}\right): \delta=$ $1.70-1.88(\mathrm{~m}, 2 \mathrm{H}), 2.00-2.10(\mathrm{~m}, 1 \mathrm{H}), 2.10-2.20(\mathrm{~m}, 1 \mathrm{H}), 2.31-2.60(\mathrm{~m}, 4 \mathrm{H}), 2.92-3.04(\mathrm{~m}, 1 \mathrm{H})$, 7.12-7.17 (d, $J=8.3 \mathrm{~Hz}, 2 \mathrm{H}), 7.25-7.32(\mathrm{~d}, J=8.5 \mathrm{~Hz}, 2 \mathrm{H}) \mathrm{ppm} .{ }^{13} \mathrm{C}-\mathrm{NMR}\left(400 \mathrm{MHz}, \mathrm{CDCl}_{3}\right)$ : $\delta=25.56(\mathrm{~s}), 32.87(\mathrm{~s}), 41.28(\mathrm{~s}), 44.27(\mathrm{~s}), 48.96(\mathrm{~s}), 128.56(\mathrm{~d}, J=86.2 \mathrm{~Hz}), 132.55$ (s), $142.96(\mathrm{~s}), 210.63$ (s) ppm.

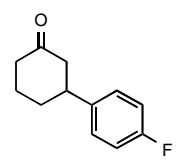

3-(4-Fluorophenyl)cyclohexanone (5ao): Eluted with $\mathrm{Hexane}_{2} \mathrm{Et}_{2} \mathrm{O}$ 9:1, obtained as colorless solid (259.5 mg, 90\% yield). HPLC: $98 \%$ ee, Chiralcel OJ-H column (n-hexane/2- 
propanol, 99.5:0.5, $1.0 \mathrm{~mL} / \mathrm{min}$ ); $t_{\mathrm{R}}: 37.5 \min$ (major), $43.5 \min$ (minor). ${ }^{1} \mathrm{H}-\mathrm{NMR}(400 \mathrm{MHz}$, $\left.\mathrm{CDCl}_{3}\right): \delta=1.70-1.88(\mathrm{~m}, 2 \mathrm{H}), 2.00-2.21(\mathrm{~m}, 2 \mathrm{H}), 2.31-2.61(\mathrm{~m}, 4 \mathrm{H}), 2.94-3.05(\mathrm{~m}, 1 \mathrm{H}), 6.97-$ $7.04(\mathrm{t}, J=8.7 \mathrm{~Hz}, 2 \mathrm{H}), 7.14-7.21(\mathrm{dd}, J=5.3$ and $8.5 \mathrm{~Hz}, 2 \mathrm{H}) \mathrm{ppm} .{ }^{13} \mathrm{C}-\mathrm{NMR}(400 \mathrm{MHz}$, $\left.\mathrm{CDCl}_{3}\right): \delta=25.59(\mathrm{~s}), 32.10(\mathrm{~s}), 41.30(\mathrm{~s}), 44.19(\mathrm{~s}), 49.26(\mathrm{~s}), 115.63(\mathrm{~d}, J=21.2 \mathrm{~Hz}), 128.18$ (d, $J=7.9 \mathrm{~Hz}), 140.25$ (d, $J=3.2 \mathrm{~Hz}), 161.76$ (d, $J=244.7 \mathrm{~Hz}), 210.81$ (s) ppm.

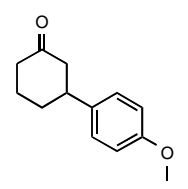

3-(4-Methoxyphenyl)cyclohexanone (5ap): Eluted with Hexane/Et $2 \mathrm{O}$ 9:1, obtained as light yellow oil (281.9 mg, 92\% yield). HPLC: 99\% ee, Chiralcel OJ-H column (n-hexane/2-propanol, 99:1, $1.0 \mathrm{~mL} / \mathrm{min}$ ); $t_{\mathrm{R}}: 45.6 \mathrm{~min}$ (major), $49.0 \mathrm{~min}$ (minor). ${ }^{1} \mathrm{H}-\mathrm{NMR}\left(400 \mathrm{MHz}, \mathrm{CDCl}_{3}\right.$ ): $\delta=$ 1.67-1.88 (m, 2H), 2.00-2.19 (m, 2H), 2.30-2.62 (m, 4H), 2.91-3.02 (m, 1H), $3.79(\mathrm{~s}, 3 \mathrm{H}), 6.83-$ $6.90(\mathrm{~d}, J=8.7 \mathrm{~Hz}, 2 \mathrm{H}), 7.11-7.17(\mathrm{~d}, J=11.6 \mathrm{~Hz}, 2 \mathrm{H}) \mathrm{ppm} .{ }^{13} \mathrm{C}-\mathrm{NMR}\left(400 \mathrm{MHz}, \mathrm{CDCl}_{3}\right): \delta=$ $25.64(\mathrm{~s}), 33.18$ (s), 41.33 (s), 44.13 (s), 49.39 (s), 55.43 (s), 114.20 (s), 127.65 (s), 136.75 (s), $158.45(\mathrm{~s}), 211.26(\mathrm{~s}) \mathrm{ppm}$.

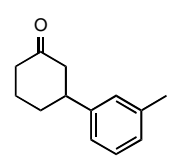

3-(3-Methylphenyl)cyclohexanone (5aq): Eluted with $\mathrm{Hexane} \mathrm{Et}_{2} \mathrm{O}$ 9:1, obtained as colorless oil (262.6 mg, 93\% yield). HPLC: 99\% ee, Chiralcel OJ-H column (n-hexane/2propanol, 99.5:0.5, $1.0 \mathrm{~mL} / \mathrm{min}$ ); $t_{\mathrm{R}}: 24.7 \mathrm{~min}$ (major), $27.9 \mathrm{~min}$ (minor). ${ }^{1} \mathrm{H}-\mathrm{NMR}(400 \mathrm{MHz}$, $\left.\mathrm{CDCl}_{3}\right): \delta=1.70-1.91(\mathrm{~m}, 2 \mathrm{H}), 2.03-2.20(\mathrm{~m}, 2 \mathrm{H}), 2.35(\mathrm{~s}, 3 \mathrm{H}), 2.32-2.63(\mathrm{~m}, 4 \mathrm{H}), 2.92-3.03$ $(\mathrm{m}, 1 \mathrm{H}), 6.99-7.08(\mathrm{~m}, 3 \mathrm{H}), 7.19-7.25(\mathrm{~m}, 1 \mathrm{H}) \mathrm{ppm} .{ }^{13} \mathrm{C}-\mathrm{NMR}\left(400 \mathrm{MHz}, \mathrm{CDCl}_{3}\right): \delta=21.68$ (s), $25.80(\mathrm{~s}), 33.05(\mathrm{~s}), 41.43(\mathrm{~s}), 44.96(\mathrm{~s}), 49.21(\mathrm{~s}), 123.77(\mathrm{~s}), 127.63(\mathrm{~d}, J=3.2 \mathrm{~Hz}), 128.79$ (s), $138.49(\mathrm{~s}), 144.57(\mathrm{~s}), 211.28(\mathrm{~s}) \mathrm{ppm}$. 


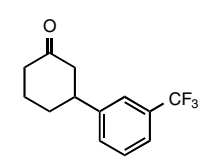

3-(3-Trifluoromethylphenyl)cyclohexanone (5ar): Eluted with Hexane/Et ${ }_{2} \mathrm{O}$ 9:1, obtained as white solid (341.6 mg, 94\% yield). HPLC: $97 \%$ ee, Chiralcel OJ-H column (n-hexane/2propanol, 99.5:0.5, $1.0 \mathrm{~mL} / \mathrm{min}$ ); $t_{\mathrm{R}}: 30.0 \mathrm{~min}$ (minor), $31.7 \mathrm{~min}$ (major). ${ }^{1} \mathrm{H}-\mathrm{NMR}(400 \mathrm{MHz}$, $\left.\mathrm{CDCl}_{3}\right): \delta=1.73-1.94(\mathrm{~m}, 2 \mathrm{H}), 2.06-2.23(\mathrm{~m}, 2 \mathrm{H}), 2.32-2.65(\mathrm{~m}, 4 \mathrm{H}), 3.02-3.13(\mathrm{~m}, 1 \mathrm{H}), 7.37-$ $7.54(\mathrm{~m}, 4 \mathrm{H}) \mathrm{ppm} .{ }^{13} \mathrm{C}-\mathrm{NMR}\left(400 \mathrm{MHz}, \mathrm{CDCl}_{3}\right): \delta=25.59$ (s), 32.77 (s), 41.24 (s), 44.69 (s), $48.83(\mathrm{~s}), 123.51(\mathrm{q}, J=3.9 \mathrm{~Hz}), 123.81(\mathrm{q}, J=3.8 \mathrm{~Hz}), 124.31(\mathrm{q}, J=272.3 \mathrm{~Hz}), 129.38(\mathrm{~s})$, $130.27(\mathrm{~d}, J=0.9 \mathrm{~Hz}), 131.24(\mathrm{q}, J=32.1 \mathrm{~Hz}), 145.39(\mathrm{~s}), 210.22(\mathrm{~s}) \mathrm{ppm} .[\alpha]_{\mathrm{D}}{ }^{25}=-0.13(\mathrm{c}=$ 1.0, $\mathrm{CHCl}_{3}$ ). HRMS calculated for $\mathrm{C}_{13} \mathrm{H}_{13} \mathrm{~F}_{3} \mathrm{O}=242.092$, found 242.092.

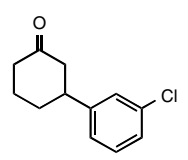

3-(3-Chlorophenyl)cyclohexanone (5as): Eluted with $\mathrm{Hexane}_{2} \mathrm{Et}_{2} \mathrm{O}$ 9:1, obtained as light yellow oil (291.1 mg, 93\% yield). HPLC: 99\% ee, Chiralcel OD-H column (n-hexane/2propanol, 99.5:0.5, $0.5 \mathrm{~mL} / \mathrm{min}$ ); $t_{\mathrm{R}}: 51.0 \mathrm{~min}$ (major), $59.5 \mathrm{~min}$ (minor). ${ }^{1} \mathrm{H}-\mathrm{NMR}$ (400 MHz, $\left.\mathrm{CDCl}_{3}\right): \delta=1.74-1.94(\mathrm{~m}, 2 \mathrm{H}), 2.06-2.27(\mathrm{~m}, 2 \mathrm{H}), 2.34-2.66(\mathrm{~m}, 4 \mathrm{H}), 2.97-3.08(\mathrm{~m}, 1 \mathrm{H}), 7.11-$ $7.16(\mathrm{~m}, 1 \mathrm{H}), 7.23-7.32(\mathrm{~m}, 3 \mathrm{H}) \mathrm{ppm} .{ }^{13} \mathrm{C}-\mathrm{NMR}\left(400 \mathrm{MHz}, \mathrm{CDCl}_{3}\right): \delta=25.59(\mathrm{~s}), 32.78(\mathrm{~s})$, 41.29 (s), 44.58 (s), 48.84 (s), 125.06 (s), 127.05 (d, J = 8.2 Hz), 130.17 (s), 134.70 (s), 146.52 (s), 210.45 (s) ppm.

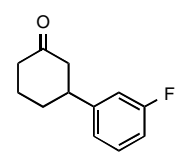

3-(3-Fluorophenyl)cyclohexanone (5at): Eluted with $\mathrm{Hexane}_{\mathrm{Et}} \mathrm{O}$ 9:1, obtained as colorless 
oil (262.4 mg, 91\% yield). HPLC: 97\% ee, Chiralcel OJ-H column (n-hexane/2-propanol, 99:1, $0.5 \mathrm{~mL} / \mathrm{min}$ ); $t_{\mathrm{R}}: 37.2 \mathrm{~min}$ (minor), $39.4 \mathrm{~min}$ (major). ${ }^{1} \mathrm{H}-\mathrm{NMR}\left(400 \mathrm{MHz}, \mathrm{CDCl}_{3}\right.$ ): $\delta=1.74-$ $1.94(\mathrm{~m}, 2 \mathrm{H}), 2.05-2.25(\mathrm{~m}, 2 \mathrm{H}), 2.35-2.67(\mathrm{~m}, 4 \mathrm{H}), 2.98-3.10(\mathrm{~m}, 1 \mathrm{H}), 6.91-7.05(\mathrm{~m}, 3 \mathrm{H}), 7.27-$ $7.35(\mathrm{~m}, 1 \mathrm{H}) \mathrm{ppm} .{ }^{13} \mathrm{C}-\mathrm{NMR}\left(400 \mathrm{MHz}, \mathrm{CDCl}_{3}\right): \delta=25.56$ (s), 32.77 (s), 41.29 (s), 44.55 (d, $J=$ $1.4 \mathrm{~Hz}), 48.87(\mathrm{~s}), 113.62(\mathrm{~d}, J=7.1 \mathrm{~Hz}), 113.83(\mathrm{~d}, J=6.8 \mathrm{~Hz}), 122.47(\mathrm{~d}, J=2.7 \mathrm{~Hz}), 130.35$ (d, $J=8.3 \mathrm{~Hz}), 147.09$ (d, $J=6.7 \mathrm{~Hz}), 162.22(\mathrm{~d}, J=245.9 \mathrm{~Hz}), 210.56$ (s) ppm.

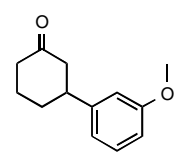

3-(3-Methoxylphenyl)cyclohexanone (5au): Eluted with Hexane/Et ${ }_{2} \mathrm{O}$ 9:1, obtained as light yellow oil (168.5 mg, 55\% yield). HPLC: 97\% ee, Chiralcel OJ-H column (n-hexane/2-propanol, 99:1, $1.0 \mathrm{~mL} / \mathrm{min}) ; t_{\mathrm{R}}: 36.3 \mathrm{~min}$ (minor), $39.7 \min$ (major). ${ }^{1} \mathrm{H}-\mathrm{NMR}\left(400 \mathrm{MHz}, \mathrm{CDCl}_{3}\right): \delta=$ 1.74-1.96 (m, 2H), 2.02-2.21 (m, 2H), 2.37-2.68 (m, 4H), 2.96-3.08 (m, 1H), $3.84(\mathrm{~s}, 3 \mathrm{H}), 6.78-$ $6.87(\mathrm{~m}, 3 \mathrm{H}), 7.25-7.32(\mathrm{~m}, 1 \mathrm{H}) \mathrm{ppm} .{ }^{13} \mathrm{C}-\mathrm{NMR}\left(400 \mathrm{MHz}, \mathrm{CDCl}_{3}\right): \delta=25.71$ (s), $32.89(\mathrm{~s})$, 41.38 (s), 44.95 (s), 49.11 (s), 55.39 (s), 111.85 (s), 112.90 (s), 119.09 (s), 129.87 (s), 146.23 (s), 160.04 (s), 211.08 (s) ppm.

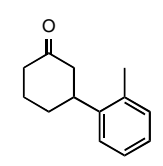

3-(2-Methylphenyl)cyclohexanone (5av): Eluted with $\mathrm{Hexane} / \mathrm{Et}_{2} \mathrm{O}$ 9:1, obtained as light yellow oil (276.8 mg, 98\% yield). HPLC: $90 \%$ ee, Chiralcel OD-H column (n-hexane/2propanol, 99.5:0.5, $0.5 \mathrm{~mL} / \mathrm{min}$ ); $t_{\mathrm{R}}: 43.2 \mathrm{~min}$ (major), $48.0 \mathrm{~min}$ (minor). ${ }^{1} \mathrm{H}-\mathrm{NMR}(400 \mathrm{MHz}$, $\left.\mathrm{CDCl}_{3}\right): \delta=1.77-1.96(\mathrm{~m}, 2 \mathrm{H}), 2.02-2.09(\mathrm{~m}, 1 \mathrm{H}), 2.17-2.26(\mathrm{~m}, 1 \mathrm{H}), 2.37(\mathrm{~s}, 3 \mathrm{H}), 2.40-2.60$ $(\mathrm{m}, 4 \mathrm{H}), 3.21-3.31(\mathrm{~m}, 1 \mathrm{H}), 7.15-7.31(\mathrm{~m}, 4 \mathrm{H}) \mathrm{ppm} .{ }^{13} \mathrm{C}-\mathrm{NMR}\left(400 \mathrm{MHz}, \mathrm{CDCl}_{3}\right): \delta=19.46$ (s), 26.00 (s), 32.23 (s), 40.52 (s), 41.50 (s), 48.55 (s), 125.28 (s), 126.63 (d, J = 4.4 Hz), 130.86 
(s), 135.30 (s), 142.49 (s), 211.37 (s) ppm.

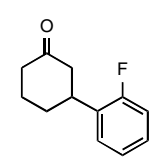

3-(2-Fluorophenyl)cyclohexanone (5aw) ${ }^{7}$ : Eluted with $\mathrm{Hexane} \mathrm{Et}_{2} \mathrm{O}$ 9:1, obtained as colorless oil (207.6 mg, 72\% yield). HPLC: 99\% ee, Chiralpak IA column (n-hexane/2-propanol, 99:1, $0.5 \mathrm{~mL} / \mathrm{min}$ ); $t_{\mathrm{R}}: 27.5 \mathrm{~min}$ (major), $29.7 \mathrm{~min}$ (minor). ${ }^{1} \mathrm{H}-\mathrm{NMR}\left(400 \mathrm{MHz}, \mathrm{CDCl}_{3}\right): \delta=$ 1.76-2.00 (m, 2H), 2.05-2.22 (m, 2H), 2.34-2.55 (m, 2H), 2.58-2.65 (d, J = 8.3 Hz, 2H), 3.30$3.40(\mathrm{~m}, 1 \mathrm{H}), 7.03-7.31(\mathrm{~m}, 4 \mathrm{H}) \mathrm{ppm} .{ }^{13} \mathrm{C}-\mathrm{NMR}\left(400 \mathrm{MHz}, \mathrm{CDCl}_{3}\right): \delta=25.53(\mathrm{~s}), 31.40(\mathrm{~s})$, 38.27 (s), 41.35 (s), 47.39 (s), 115.84 (d, $J=22.6 \mathrm{~Hz}), 124.49$ (d, $J=3.5 \mathrm{~Hz}), 127.77$ (d, $J=4.8$ $\mathrm{Hz}), 128.31(\mathrm{~d}, J=8.4 \mathrm{~Hz}), 131.15(\mathrm{~d}, J=13.9 \mathrm{~Hz}), 160.68$ (d, $J=245.7 \mathrm{~Hz}), 211.17$ (s) ppm.

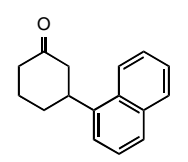

3-(1-Naphthyl)cyclohexanone (5ax): Eluted with Hexane/Et $2 \mathrm{O}$ 9:1, obtained as white solid (333.1 mg, 99\% yield). HPLC: 90\% ee, Chiralcel OD-H column (n-hexane/2-propanol, 95:5, 0.5 $\mathrm{mL} / \mathrm{min}$ ); $t_{\mathrm{R}}: 42.8 \mathrm{~min}$ (minor), $62.5 \mathrm{~min}$ (major). ${ }^{1} \mathrm{H}-\mathrm{NMR}\left(400 \mathrm{MHz}, \mathrm{CDCl}_{3}\right.$ ): $\delta=1.86-2.08$ (m, 2H), 2.15-2.30 (m, 2H), 2.41-2.82 (m, 4H), 3.81-3.92 (m, 1H), 7.38-7.57 (m, 4H), 7.73-7.79 $(\mathrm{d}, J=8.1 \mathrm{~Hz}, 1 \mathrm{H}), 7.85-7.91(\mathrm{~d}, J=8.9 \mathrm{~Hz}, 1 \mathrm{H}), 8.01-8.07(\mathrm{~d}, J=8.4 \mathrm{~Hz}, 1 \mathrm{H}) \mathrm{ppm} .{ }^{13} \mathrm{C}-\mathrm{NMR}$ (400 MHz, $\left.\mathrm{CDCl}_{3}\right): \delta=25.80$ (s), 32.53 (s), 39.61 (s), 41.66 (s), 48.79 (s), 122.78 (d, $J=27.1$ Hz), 125.79 (d, $J=10.8 \mathrm{~Hz}), 126.42$ (s), 127.47 (s), 129.38 (s), 131.13 (s), 134.20 (s), 140.27 (s), 211.38 (s) ppm.

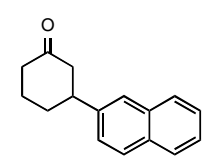

3-(2-Naphthyl)cyclohexanone (5ay): Eluted with Hexane/Et $\mathrm{H}_{2} \mathrm{O}$ 9:1, obtained as white solid 
(299.4 mg, 89\% yield). HPLC: 96\% ee, Chiralcel OD-H column (n-hexane/2-propanol, 98:2, 0.5 $\mathrm{mL} / \mathrm{min}$ ); $t_{\mathrm{R}}: 64.9 \mathrm{~min}$ (major), $75.4 \mathrm{~min}$ (minor). ${ }^{1} \mathrm{H}-\mathrm{NMR}$ (400 $\mathrm{MHz}, \mathrm{CDCl}_{3}$ ): $\delta=1.77-2.05$ (m, 2H), 2.13-2.26 (m, 2H), 2.37-2.56 (m, 2H), 2.60-2.75 (m, 2H), 3.13-3.26 (m, 1H), 7.35-7.40 $(\mathrm{d}, J=8.5 \mathrm{~Hz}, 1 \mathrm{H}), 7.42-7.51(\mathrm{~m}, 2 \mathrm{H}), 7.65(\mathrm{~s}, 1 \mathrm{H}), 7.53-7.87(\mathrm{~m}, 3 \mathrm{H}) \mathrm{ppm} .{ }^{13} \mathrm{C}-\mathrm{NMR}(400$ $\left.\mathrm{MHz}, \mathrm{CDCl}_{3}\right): \delta=25.75(\mathrm{~s}), 32.96(\mathrm{~s}), 41.47(\mathrm{~s}), 45.03(\mathrm{~s}), 49.08(\mathrm{~s}), 124.96(\mathrm{~s}), 125.69(\mathrm{~d}, J=$ $32.9 \mathrm{~Hz}), 126.41$ (s), 127.86 (d, J = 7.3 Hz), 128.58 (s), 132.61 (s), 133.49 (s), 141.97 (s), 211.11 (s) ppm.

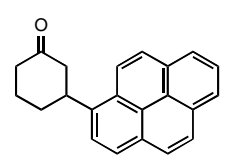

3-(1-Pyrenyl)cyclohexanone (5az): Eluted with Hexane/EtOAc 3:2, obtained as beige solid (402.8 mg, 90\% yield). HPLC: 95\% ee, Chiralcel OD-H column (n-hexane/2-propanol, 90:10, $0.5 \mathrm{~mL} / \mathrm{min}$ ); $t_{\mathrm{R}}: 30.5 \mathrm{~min}$ (major), $34.0 \mathrm{~min}$ (minor). ${ }^{1} \mathrm{H}-\mathrm{NMR}\left(400 \mathrm{MHz}, \mathrm{CDCl}_{3}\right.$ ): $\delta=1.94-$ $2.38(\mathrm{~m}, 4 \mathrm{H}), 2.47-2.68(\mathrm{~m}, 2 \mathrm{H}), 2.75-2.90(\mathrm{~m}, 2 \mathrm{H}), 4.11-4.23(\mathrm{~m}, 1 \mathrm{H}), 7.95-8.31(\mathrm{~m}, 9 \mathrm{H}) \mathrm{ppm}$.

${ }^{13} \mathrm{C}-\mathrm{NMR}\left(400 \mathrm{MHz}, \mathrm{CDCl}_{3}\right.$ ): $\delta=26.01$ (s), 32.98 (s), 40.14 (s), 41.67 (s), 49.30 (s), 122.35 (s), 126.89 (s), 123.06 (s), 125.18 (s), 125.22 (s), 125.33 (s), 125.44 (s), 126.21 (s), 127.35 (s), 127.59 (s), 128.03 (s), 128.09 (s), 130.26 (s), 130.95 (s), 131.67 (s), 137.95 (s), 211.22 (s) ppm. $[\alpha]_{\mathrm{D}}^{25}=-0.89\left(\mathrm{c}=1.0, \mathrm{CHCl}_{3}\right)$. HRMS calculated for $\mathrm{C}_{22} \mathrm{H}_{18} \mathrm{O}=298.136$, found 298.136.

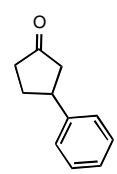

3-Phenylcyclopentanone (5bl): Eluted with $\mathrm{Hexane}_{\mathrm{Et}} \mathrm{O}$ 9:1, obtained as colorless oil (237.9 mg, 99\% yield). HPLC: 96\% ee, Chiralcel OB column (n-hexane/2-propanol, 99.5:0.5, $1.0 \mathrm{~mL} / \mathrm{min}$ ); $t_{\mathrm{R}}: 34.5 \mathrm{~min}$ (major), $39.3 \mathrm{~min}$ (minor). ${ }^{1} \mathrm{H}-\mathrm{NMR}\left(400 \mathrm{MHz}, \mathrm{CDCl}_{3}\right.$ ): $\delta=1.97-$ $2.11(\mathrm{~m}, 1 \mathrm{H}), 2.28-2.57(\mathrm{~m}, 4 \mathrm{H}), 2.66-2.77(\mathrm{~m}, 1 \mathrm{H}), 3.41-3.53(\mathrm{~m}, 1 \mathrm{H}), 7.26-7.32(\mathrm{~m}, 3 \mathrm{H}), 7.36-$ 
7.42 (m, 2H) ppm. ${ }^{13} \mathrm{C}-\mathrm{NMR}\left(400 \mathrm{MHz}, \mathrm{CDCl}_{3}\right): \delta=31.39$ (s), 39.05 (s), 42.42 (s), 45.99 (s), $126.92(\mathrm{~s}), 128.88(\mathrm{~s}), 143.27(\mathrm{~s}), 218.51$ (s) ppm.

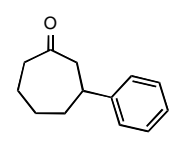

3-Phenylcycloheptanone $(\mathbf{5 c l})^{\mathbf{7 , 8}}$ : Eluted with Hexane/Et ${ }_{2} \mathrm{O}$ 9:1, obtained as colorless oil (276.8 mg, 98\% yield). HPLC: 66\% ee, Chiralcel OD-H column (n-hexane/2-propanol, 98:2, 0.5 $\mathrm{mL} / \mathrm{min}$ ); $t_{\mathrm{R}}: 21.3 \mathrm{~min}$ (major), $22.9 \mathrm{~min}$ (minor). ${ }^{1} \mathrm{H}-\mathrm{NMR}$ (400 $\mathrm{MHz}, \mathrm{CDCl}_{3}$ ): $\delta=1.43-1.58$ (m, 1H), 1.66-1.82 (m, 2H), 1.96-2.14 (m, 3H), 2.56-2.71 (m, 3H), 2.86-2.99 (m, 2H), 7.16-7.24 (m, 3H), 7.27-7.33 (m, 2H) ppm. ${ }^{13} \mathrm{C}-\mathrm{NMR}\left(400 \mathrm{MHz}, \mathrm{CDCl}_{3}\right): \delta=24.38$ (s), 29.45 (s), 39.4 (s), 42.95 (s), 44.14 (s), 51.46 (s), 126.58 (d, J = 6.8 Hz), 128.85 (s), 147.512 (s), 213.58 (s) ppm.

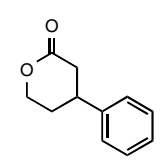

4-Phenyl-tetrahydro-2H-pyran-2-one (5dl): Eluted with Hexane/EtOAc 4:1, obtained as light brown viscous oil (259.0 mg, 98\% yield). HPLC: 91\% ee, Chiralcel OD-H column (nhexane/2-propanol, 90:10, $0.5 \mathrm{~mL} / \mathrm{min}$ ); $t_{\mathrm{R}}: 59.0 \mathrm{~min}$ (minor), $61.6 \mathrm{~min}$ (major). ${ }^{1} \mathrm{H}-\mathrm{NMR}$ (400 $\left.\mathrm{MHz}, \mathrm{CDCl}_{3}\right): \delta=1.99-2.21(\mathrm{~m}, 2 \mathrm{H}), 2.46-2.57(\mathrm{dd}, J=18.1$ and $9.1 \mathrm{~Hz}, 1 \mathrm{H}), 2.84-2.95(\mathrm{ddd}, J$ $=18.2$ and $6.1 \mathrm{~Hz}, J=2.2 \mathrm{~Hz}, 1 \mathrm{H}), 3.19-3.30(\mathrm{~m}, 1 \mathrm{H}), 4.42-4.68(\mathrm{~m}, 2 \mathrm{H}), 7.09-7.50(\mathrm{~m}, 5 \mathrm{H})$ ppm. ${ }^{13} \mathrm{C}-\mathrm{NMR}\left(400 \mathrm{MHz}, \mathrm{CDCl}_{3}\right): \delta=30.21$ (s), $37.42(\mathrm{~s}), 37.48$ (s), 68.57 (s), 126.43 (s), 127.18(s), $128.91(\mathrm{~s}), 142.75(\mathrm{~s}), 170.62(\mathrm{~s}) \mathrm{ppm}$.

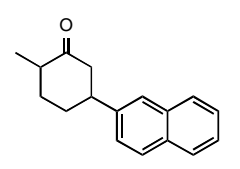

3-(2-Naphthyl)-6-methyl-cyclohexanone (5ey): ${ }^{7}$ Eluted with Hexane/Et ${ }_{2} \mathrm{O}$ 9:1, two 
diastereoisomers (cis and trans) in a 1:1 ratio, both obtained as white solids $(178+178 \mathrm{mg}, 99 \%$ yield). HPLC: Chiralcel OD-H column (n-hexane/2-propanol, 99.5:0.5, $1.0 \mathrm{~mL} / \mathrm{min}$ ); first diastereoisomer eluted from flash chromatography, 94\% ee; $t_{\mathrm{R}}: 52.7 \mathrm{~min}$ (major), $77.9 \mathrm{~min}$ (minor); second diastereoisomer eluted from flash chromatography, 94\% ee; $t_{\mathrm{R}}: 37.1 \mathrm{~min}$ (major), 58.4 min (minor). First diastereoisomer eluted from flash chromatography: ${ }^{1} \mathrm{H}-\mathrm{NMR}$ $\left(400 \mathrm{MHz}, \mathrm{CDCl}_{3}\right): \delta=1.10-1.14(\mathrm{~d}, J=6.5 \mathrm{~Hz}, 3 \mathrm{H}), 1.49-1.62(\mathrm{~m}, 1 \mathrm{H}), 1.98-2.30(\mathrm{~m}, 3 \mathrm{H})$, 2.47-2.74 (m, 3H), 3.10-3.20 (m, 1H), 7.35-7.40 (dd, $J=1.8$ and 8.5 Hz, 1H), 7.42-7.51 (m, 2H), $7.65(\mathrm{~s}, 1 \mathrm{H}), 7.77-7.86(\mathrm{~m}, 3 \mathrm{H}) \mathrm{ppm} .{ }^{13} \mathrm{C}-\mathrm{NMR}\left(400 \mathrm{MHz}, \mathrm{CDCl}_{3}\right): \delta=14.57$ (s), 33.43 (s), 35.32 (s), 44.92 (s), 46.16 (s), 49.30 (s), 124.79 (s), 125.44 (s), 125.77 (s), 126.33 (s), 127.81 (d, $J=5.3 \mathrm{~Hz}), 128.48(\mathrm{~s}), 132.58(\mathrm{~s}), 133.75(\mathrm{~s}), 141.92(\mathrm{~s}), 212.06(\mathrm{~s}) \mathrm{ppm} .[\alpha]_{\mathrm{D}}^{25}=-0.17(\mathrm{c}=$ 1.0, $\mathrm{CHCl}_{3}$ ). HRMS calculated for $\mathrm{C}_{17} \mathrm{H}_{18} \mathrm{O}=238.136$, found 238.136.

Second diastereoisomer eluted from flash chromatography: ${ }^{1} \mathrm{H}-\mathrm{NMR}\left(400 \mathrm{MHz}, \mathrm{CDCl}_{3}\right): \delta=$ $1.18-1.22(\mathrm{~d}, J=7.0 \mathrm{~Hz}, 3 \mathrm{H}), 1.59-1.70(\mathrm{~m}, 1 \mathrm{H}), 1.90-2.00(\mathrm{~m}, 1 \mathrm{H}), 2.10-2.18(\mathrm{~m}, 2 \mathrm{H}), 2.51-$ $2.70(\mathrm{~m}, 2 \mathrm{H}), 2.85-2,94(\mathrm{~m}, 1 \mathrm{H}), 3.41-3.50(\mathrm{~m}, 1 \mathrm{H}), 7.33-7.38(\mathrm{dd}, J=1.8$ and $8.5 \mathrm{~Hz}, 1 \mathrm{H})$, 7.42-7.50 (m, 2H), $7.62(\mathrm{~s}, 1 \mathrm{H}), 7.78-7.84(\mathrm{~m}, 3 \mathrm{H}) \mathrm{ppm} .{ }^{13} \mathrm{C}-\mathrm{NMR}\left(400 \mathrm{MHz}, \mathrm{CDCl}_{3}\right): \delta=$ 15.93 (s), 29.70 (s), 30.76 (s), 43.14 (s), 44.50 (s), 45.04 (s), 125.30 (s), 125.69 (s), 125.85 (s), 126.21 (s), 127.61 (s), 127.88 (s), 128.27 (s), 132.25 (s), 133.52 (s), 141.79 (s), 214.03 (s) ppm. $[\alpha]_{\mathrm{D}}^{25}=+0.01\left(\mathrm{c}=1.0, \mathrm{CHCl}_{3}\right)$. HRMS calculated for $\mathrm{C}_{17} \mathrm{H}_{18} \mathrm{O}=238.136$, found 238.136

\section{Attempts for epimerization of 5ey:}

1) Basic conditions: Pure cis-5ey (94\% ee) or a racemic mixture of the four possible, ${ }^{9}$ diastereoisomers of 5ey (100mg, $0.4196 \mathrm{mmol})$ was stirred for 48 hours in a solution of $\mathrm{NaOMe} / \mathrm{MeOH}(2.1 \mathrm{ml}, 0.8392 \mathrm{mmol})$ at room temperature. The reaction mixture was diluted with ether, washed with concentrated $\mathrm{NH}_{4} \mathrm{Cl}$, concentrated $\mathrm{NaHCO}_{3}$, and brine. The organic phase was dried with $\mathrm{MgSO}_{4}$, filtered and concentrated to give the product in quantitative yield. 
HPLC analysis showed no changes in substrate composition. $\mathrm{NaOEt} / \mathrm{EtOH}$ and refluxing conditions were also tried without success affording the same results.

2) Acidic conditions: Pure cis-5ey (94\% ee) or a racemic mixture of the four possible diastereoisomers of 5ey $(100 \mathrm{mg}, 0.4196 \mathrm{mmol})$ was stirred for 48 hours in a solution of $\mathrm{HCl} / \mathrm{MeOH}$ (2 drops of $\mathrm{HCl} 5 \mathrm{M}$ in $2 \mathrm{ml} \mathrm{MeOH}$ ) at room temperature. The reaction mixture was diluted with ether, washed with concentrated $\mathrm{NH}_{4} \mathrm{Cl}$, concentrated $\mathrm{NaHCO}_{3}$, and brine. The organic phase was dried with $\mathrm{MgSO}_{4}$, filtered and concentrated to give the product in quantitative yield. HPLC analysis showed no changes in substrate composition. Refluxing conditions were also tried without success affording the same results.

\section{References:}

1 Boiteau, J. G.; Imbos R., Minnaard A. J., Feringa B. L., Org. Lett. 2003, 5, 681.

2 Chen, Q.; Kuriyama, M.; Soeta, T.; Hao, X.; Yamada, K.; Tomioka, K. Org. Lett. 2005, 7, 4439.

3 Vandyck, C.; Matthys, B.; Willen, M.; Robeyns, K.; Van Meervelt, L.; Van der Eycken, J. Org. Lett. 2006, 8, 363.

4 Stemmler, R. T.; Bolm C. J. Org. Chem. 2005, 70, 9925.

5 Marques, F. A.; Lenz, C. A.; Simonelli, F.; Sales Maria, B. H. L. N.; Vellasco, A. P.; Eberlin M. N. J. Nat. Prod. 2004, 67, 1939.

6 Clayden, J.; Kubinski, P. M.; Sammiceli, F.; Helliwell, M.; Diorazio, L. Tetrahedron 2004, $60,4387$.

7 Run with 2 eq. of boronic acid and $3 \mathrm{~mol} \%$ of $\mathrm{Rh}$.

8 Distillation of 2-cyclohepten-1-one instead of flash chromatography might give purer starting material and better ee values in catalysis: Otomaru, Y.; Senda, T.; Hayashi, T. Org. Lett. 2004, 6, 3357. 
9 Prepared from an equimolar amount of $\left[\left\{\left(P, R_{S}, R_{S}\right)-p \text {-tol-BINASO }\right\} \mathrm{RhCl}\right]_{2}(15 \mathrm{mg}, 0.0113$ $\mathrm{mmol})$ and $\left[\left\{\left(M, S_{S}, S_{S}\right)-p\right.\right.$-tol-BINASO $\} \mathrm{RhCl}_{2}(15 \mathrm{mg}, 0.0113 \mathrm{mmol})$ following the same procedure as for $\mathbf{5 e y}$. 